\title{
MEASURING THE MASSES OF COMPACT OBJECTS IN LOW-MASS X-RAY BINARIES
}

\author{
Dawn M. Gelino ${ }^{1}$
}

Low-mass X-ray binaries (LMXBs) contain compact, black hole (BH) or neutron star (NS) primaries, and cool, low-mass secondary stars. We measure the orbital inclination of the system in quiescence by modeling infrared (IR) ellipsoidal variations from the secondary star in order to determine the compact object mass. I present our results for a few LMXBs, including the first $\mathrm{BH}$ that appears to conclusively fall in the $3-5 M_{\odot}$ range.

Compact object masses have significant consequences for the physics of supernova explosions, $\mathrm{BH}$ formation mechanisms, and even the expected event rate for gravitational radiation detectors. Even with these limited statistics (19 BH, 17 NS masses), there is a lack of BHs with masses between 1.4 and 5 $\mathrm{M}$. .). This observational finding does not agree with the detailed prediction of a continuous distribution with the largest number of $\mathrm{BHs}$ just above the NS mass limit, and decreasing for higher masses (Fryer \& Kalogera 2001, ApJ, 554, 548).

Ellipsoidal variations result from the tidal and rotational distortions of the Roche lobe-filling secondary star, as well as its non-uniform surface brightness distribution. The amplitude of the ellipsoidal variations is a strong function of $i$, and can therefore be modeled to find the inclination of the system. IR variations are much less contaminated/diluted by accretion disk light than optical variations. It is important to accurately account for this extra light since dilution of the variations results in an underestimate of $i$, and an overestimate of $\mathrm{M}_{1}$. Fig. 1 shows KPNO $4 \mathrm{~m}$ IR light curves and WD98 models for the faint NS system, XTE J2123-058.

Table 1 presents some orbital inclination angles, compact object masses, and distances derived with our IR observing and modeling technique, while Table 2 presents the secondary star masses, orbital separations, and secondary star Roche lobe volume radii. XTE J2123-058 appears to harbor an overmassive neutron star. however, higher precision photometry and radial velocity measurements are needed to further constrain the mass of this faint

\footnotetext{
${ }^{1}$ Center for Astrophysics and Space Sciences, Iniversity of California San Diego, MSC 0424, 9500 Gilman Drive, La Jolla, CA 92093-0424 (dgelino@ucsd.edu).
}

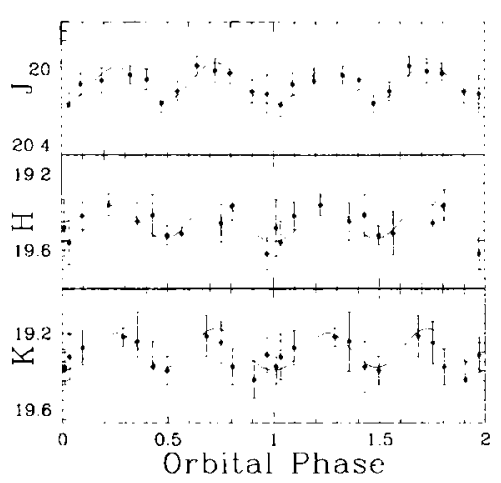

Fig. 1. XTE J2123-058 $J$-, $H$-, \& $K$-band light curves (points) and WD98 models (lines). Error bars are $1 \sigma$. The data of this very faint ( $J=20.12 \mathrm{mag}$ ) source show no obvious signs of IR contamination. With $q=0.36$ (Tomsick et al. 2002, ApJ, 581, 570), a $\chi^{2}$ minimization finds the best fit model to have $i=65 \pm 4^{\circ}$.

TABLE 1

\section{IR DERIVED LMXB PARAMETERS}

\begin{tabular}{lclc}
\hline Object & $i\left(^{\circ}\right)$ & $\mathrm{M}_{\mathbf{1}}\left(\mathrm{M}_{\odot}\right)$ & $\mathrm{d}(\mathrm{kpc})$ \\
\hline XTE J2123-058 & $65 \pm 4$ & $1.71 \pm 0.73$ & $7.92 \pm 2.21$ \\
GRO J0422+32 $^{\mathrm{b}}$ & $42 \pm 2$ & $3.97 \pm 0.95$ & $2.49 \pm 0.30$ \\
GRS 1009-45 & $67 \pm 3$ & $5.24 \pm 0.60$ & $3.82 \pm 0.27$ \\
GRS 1124-68 & $54 \pm 1.5$ & $7.24 \pm 0.70$ & $5.89 \pm 0.26$ \\
GS 2000+25 & $64 \pm 1.5$ & $7.70 \pm 0.66$ & $2.29 \pm 0.15$ \\
A0620-00 & $41 \pm 3$ & $\mathbf{1 1 . 0 \pm 1 . 9}$ & $1.16 \pm 0.11$ \\
${ }^{\mathrm{a}}$ This work & \multicolumn{3}{l}{} \\
${ }^{\mathrm{b}}$ Gelino \& Harrison 2003, ApJ, in Press \\
${ }^{\mathrm{C}}$ Gelino \& Harrison 2002, BAAS, 34, 654 \\
dGelino, Harrison, \& McNamara 2001, AJ, 122, 971 \\
${ }^{\mathrm{e}}$ Gelino 2001, PhD Thesis, NMSU \\
${ }^{\mathrm{f}}$ Gelino, Harrison, \& Orosz 2001, AJ, 122, 2668
\end{tabular}

TABLE 2

IR DERIVED LMXB PARAMETERS ${ }^{\mathrm{a}}$

\begin{tabular}{lccc}
\hline Object & $\mathrm{M}_{2}\left(\mathrm{M}_{\odot}\right)$ & $a\left(\mathrm{R}_{\odot}\right)$ & $\mathrm{R}_{L_{2}}\left(\mathrm{R}_{\odot}\right)$ \\
\hline XTE J2123-058 & $0.62 \pm 0.59$ & $2.20 \pm 0.37$ & $0.65 \pm 0.18$ \\
GRO J0422+32 & $0.46 \pm 0.31$ & $2.45 \pm 0.24$ & $0.53 \pm 0.16$ \\
GRS 1009-45 & $0.72 \pm 0.16$ & $3.30 \pm 0.13$ & $0.75 \pm 0.06$ \\
GRS 1124-68 & $0.94 \pm 0.20$ & $4.85 \pm 0.15$ & $1.08 \pm 0.05$ \\
GS 2000+25 & $0.32 \pm 0.11$ & $4.14 \pm 0.13$ & $0.66 \pm 0.08$ \\
A0620-00 & $0.68 \pm 0.18$ & $4.47 \pm 0.27$ & $0.80 \pm 0.07$ \\
TReferences are the same as in Table 1.
\end{tabular}

system. GRO J0422+32 contains the lowest mass black hole measured, and the first to fall in the 3-5 $\mathrm{M}_{2}$ range. More accurate masses are needed to test the true distribution of $\mathrm{BH}$ masses. 